\title{
Treadmill Test to Detect Stress Induced Ischemic Heart Disease in Type 2 Diabetes Mellitus Patients Asymptomatic for CAD: A Hospital Based Cross-sectional Study in Rural Population of Central India
}

\author{
Anurag S Lavekar* and Harsha R Salkar
}

Department of Medicine, NKP Salve Institute of Medical Sciences and Research Centre, Maharashtra, India

\begin{abstract}
Background: Progressive Coronary Artery Disease (CAD) remains asymptomatic in many cases of type 2 diabetes mellitus (DM) and this makes diagnosis difficult. Routine screening of type 2 DM for silent ischemia with electrocardiography (ECG) remains controversial as majority of patients present with normal ECG. This study aims at using Treadmill Test (TMT) to determine the percentage of ischemic heart disease (IHD) in diabetic patients, having normal resting ECG and 2D Echo, in a rural set up of Central India. Moreover, the interest is to determine the risk factors for IHD in patients who underwent TMT.

Materials and methods: One hundred sixty one type 2 DM patients in the age range 30-60 years with normal baseline ECG and resting Echo were screened by TMT. The data on demographics, biochemical tests and micro vascular complications was obtained for each patient to determine the possible risk factors of IHD in this study population.

Results: Thirty four out of 161 patients $(21.1 \%)$ were found positive for exercise induced ischemia. Of these, 27 underwent exercise stress echo immediately after TMT and the presence of RWMA was confirmed in 20 patients. Age, duration of DM and family history of IHD were identified as major covariates in the study. The parameters like $\mathrm{HbA}_{1} \mathrm{C}$ (glycosylated hemoglobin), total cholesterol (TC), triglycerides (TG) and low density lipoprotein (LDL) showed marginal increase of odds in favor of TMT positive result after adjusting for covariates. Nephropathy and retinopathy were associated with silent ischemia in the study population exposed to TMT.

Discussion: The study group belonged to lower socioeconomic group with mostly moderate to heavy working life style. Patients had normal resting echo, but when subjected to TMT, a significant proportion of cases were positive for silent ischemia, which otherwise would have been unnoticed. We believe that TMT remains the cornerstone for screening the population at risk in the rural sector where angiography is not readily available.
\end{abstract}

Keywords: Type 2 diabetes mellitus; 2D Echo; Microvascular complications; Ischemic heart disease

\section{Introduction}

Type 2 diabetes mellitus (DM) has got a distinct association with coronary artery disease (CAD); and diabetic patients have 2-4 fold higher risk of developing CAD than people without DM [1]. However, progressive coronary artery disease remains asymptomatic in many cases of type 2 diabetes and this makes diagnosis difficult at proper time [2]. Routine screening of type 2 diabetics for asymptomatic coronary artery disease with ECG remains controversial, as majority of them present with normal ECG [3]. Many studies have been published showing relationship between DM and silent ischemia; but there are hardly any studies targeting rural population, especially from Central India, where the demographics is totally different than urban settings. We present here a study showing relationship between type $2 \mathrm{DM}$ and silent ischemia in patients representing this population, arriving in a tertiary health care hospital located in the outskirts of Nagpur, India. The interest here is to determine the proportion of silent ischemia in patients having type $2 \mathrm{DM}$, who are asymptomatic for CAD and have normal resting ECG and 2D Echo using TMT. Moreover, the interest is to determine the predictors of silent ischemia in such study population who underwent TMT.

\section{Materials and Methods}

\section{Study population}

In view of the above interest, a protocol was designed and presented to the Institutional Ethical Committee. Type $2 \mathrm{DM}$ patients in the age range of 30-60 years of either sex attending the tertiary care rural hospital during period October 2010 to April 2012 were targeted for the study. The reason for focusing on this age group was that, patients with age less than 30 years may have type 1 diabetes mellitus and patient with age more than 60 may not be able to walk on treadmill. People from villages and small towns in the radius of about $100 \mathrm{~km}$ visit the hospital covering eastern region of Vidarbha, southern part of Madhya Pradesh and western region of Chhattisgarh. It was decided to screen all type $2 \mathrm{DM}$ patients attending the hospital during this period and following the inclusion criteria. Informed consent was taken of all patients was taken. Patients following the above age criterion, having normal baseline ECG and with or without hypertension were included in the study. Excluded patients were those with history of CAD or any

*Corresponding author: Anurag S. Lavekar, Department of Medicine, NKP Salve Institute of Medical Sciences and Research Centre, Hingna, Nagpur, Maharashtra India, Tel: +91 9673338292; E-mail: anuraglavekar@gmail.com

Received January 18, 2013; Accepted February 16, 2013; Published February 20, 2013

Citation: Lavekar AS, Salkar HR (2013) Treadmill Test to Detect Stress Induced Ischemic Heart Disease in Type 2 Diabetes Mellitus Patients Asymptomatic fo CAD: A Hospital Based Cross-sectional Study in Rural Population of Central India. J Diabetes Metab 4: 244. doi:10.4172/2155-6156.1000244

Copyright: (c) 2013 Lavekar AS, et al. This is an open-access article distributed under the terms of the Creative Commons Attribution License, which permits unrestricted use, distribution, and reproduction in any medium, provided the original author and source are credited. 
Citation: Lavekar AS, Salkar HR (2013) Treadmill Test to Detect Stress Induced Ischemic Heart Disease in Type 2 Diabetes Mellitus Patients Asymptomatic for CAD: A Hospital Based Cross-sectional Study in Rural Population of Central India. J Diabetes Metab 4: 244. doi:10.4172/2155-6156.1000244

Page 2 of 5

other congenital heart disease, arrhythmias, overt diabetic autonomic neuropathy, proliferative diabetic retinopathy, chronic kidney disease (CKD), resting $2 \mathrm{D}$ Echo suggestive of regional wall motion abnormality (RWMA), and hemodynamically unstable patients. The patients were mostly from lower middle class, and were either farmers, or skilled and unskilled workers from small industry. Most of them had moderate to heavy working life style.

\section{Study design}

In the present hospital based cross-sectional study, a structured pre-tested questionnaire was developed to seek information on demographics of patient, duration of DM at the time of hospital visit, history of $\mathrm{DM} / \mathrm{IHD}$, dietary habits, smoking and alcohol intake, socioeconomic status etc. The questionnaire also included columns to record observations on various investigational parameters for each patient.

\section{Biochemical tests}

$\mathrm{HbA}_{1} \mathrm{C}$ and fasting lipid profile was done. Lipid parameters were assessed by the cell counter using E Selectra Merg machine. Dyslipidemia was defined as TC $\geq 240 \mathrm{mg} / \mathrm{dl}$, TG $\geq 150 \mathrm{mg} / \mathrm{dl}$, LDL $\geq$ $100 \mathrm{mg} / \mathrm{dl}$ and high density lipoprotein $(\mathrm{HDL})<40 \mathrm{mg} / \mathrm{dl}$ in men and $<50 \mathrm{mg} / \mathrm{dl}$ in women [4]

\section{D Echo}

2D Echocardiography of all patients included in the study was done on Toshiba Nemio XG SSA - 580A machine with a $3 \mathrm{MHz}$ cardiac probe. Patients were screened in supine and/or left lateral position. Parasternal short axis, parasternal long axis, apical four chamber, apical two chamber and sub-costal views along with $\mathrm{M}$-mode echo and doppler pulse wave echo were used to assess regional wall motion abnormality (RWMA), and left ventricular systolic and diastolic function. Left ventricular ejection fraction (LVEF) was calculated by using Modified Simpson's rule. It is the measure of left ventricular volume in biplane method of discs. The principle is that the total left ventricular volume is calculated from the summation of a stack of elliptical discs. The left ventricular volumes from apical 4 and/or apical 2 chamber views are measured, tracing the endocardial border of left ventricle. The Echocardiography machine automatically gives the volume of cavity. Presence or absence of RWMA was determined. Resting 2D echo was done considering that ischemic changes can be picked up on $2 \mathrm{D}$ echo even before the appearance of patient's symptoms of IHD. TMT positive cases were subjected to exercise stress echo for confirmation of RWMA.

\section{Microvascular complications}

Diabetic retinopathy was diagnosed by a professional ophthalmologist by fundoscopic examination. Non-proliferative diabetic retinopathy (NPDR) was described when micro-aneurysms, hemorrhages, intra-retinal microvascular abnormalities (IRMA) and venous bleeding were present. Total urine sample within 24 hour was collected in a clean glass container free from detergent. Before test specimen was tested within half hours of collection. Turbidimetric immunoassay method (AGAPPE diagnostics India) for determination of micro-albuminuria and macro-albuminuria was used. Microalbuminuria was defined as $24 \mathrm{hrs}$ urinary proteins of $30-300 \mu \mathrm{g} / \mathrm{ml}$ and macro-albuminuria was defined as 24 hrs urinary proteins 300 $3000 \mu \mathrm{g} / \mathrm{ml}$. [4]. Diabetic neuropathy was diagnosed on the basis of patient's symptoms, e.g. tingling and numbness, paresthesia and clinical examination (depressed or absent deep tendon reflexes, loss of pinprick sensation, loss of vibration sensation in toes). Nerve conduction velocity test (NCV) was not done.

\section{Treadmill test}

TMT was performed with BPL Dynatrac ver 3.8 software, using the Bruce protocol. The Bruce multistage maximal treadmill protocol has 3 minutes period to allow achievement of steady state before workload is increased. A positive response, i.e. ischemic heart disease was defined as ST segment depression $>1 \mathrm{~mm}$ horizontal or down sloping. Hypertensive TMT response was defined as systolic $\mathrm{BP}>200 \mathrm{mmHg}$. Targeted heart rate (THR) was calculated by using formula $\mathrm{THR}=220$ - age (in years)

\section{Statistical methods}

Descriptive statistics on demographic characteristics of patients were obtained according to TMT findings. The statistics included mean and standard deviation for continuous variables and frequencies (percentage) for categorical variables. Student's $t$-test for independent samples was used to determine statistical significance of difference in the mean values of continuous variable between TMT positive and negative groups. Chi-square test was used to determine if the frequency distribution across the levels of categorical variables has any statistical association with TMT findings. Odds ratio for each lipid parameter was obtained using univariate logistic regression with lipid parameter as independent and TMT outcome as dependent variable. For microvascular complications, the odds ratios were obtained based on contingency tables. The odds ratio were also obtained after adjusting for covariates (demographic variables) using multivariate logistic regression. Model fit was evaluated using Hosmer-Lemeshow test. Statistical significance was evaluated at $5 \%$ level and all the analysis was performed using $R 2.15$ package (The R Foundation for Statistical Computing).

\section{Results}

Out of 161 patients, $34(21.1 \%)$ patients were TMT positive, while 90 patients were negative (55.9\%) and $37(22.9 \%)$ were inconclusive for IHD based on TMT test. Out of 34 TMT positive cases, 27 underwent stress echo immediately after the completion of TMT. In remaining 7 patients, stress echo could not be done because of unwillingness of patients to undergo stress echo test. From these 27 patients, presence of regional wall motion abnormality was confirmed in 20 patients $(74 \%)$ indicating that TMT was false positive in remaining 7 (26\%) patients.

\section{Demographic variables}

The mean age of subjects in TMT positive group ( $53.56 \pm 7.41$ years) was significantly higher than that of TMT negative group $(48.71 \pm 8.72$ yrs) with a $\mathrm{P}$ value of 0.0023 (Table 1). The proportion of male subjects was statistically insignificant across the groups. The mean duration of diabetes in TMT positive group (74.79 \pm 48.2 months) was significantly higher than that of TMT negative group (47.41 \pm 31.66 months) with a P value of 0.0007 . Further, family history of IHD showed significant association with TMT results with a $\mathrm{P}$ value of $0.0136(P<0.05)$. This was due to higher proportion of TMT positive cases (50\%) presenting with family history of above systemic illness. Personal history (smoking, alcohol), dietary habits and life style did not show any association with TMT results as indicated by their respective P-values of $0.8759,0.6595$ and 0.4213 . Also, mean BMI was statistically insignificant between the two groups with $\mathrm{P}=0.1364$. 
Citation: Lavekar AS, Salkar HR (2013) Treadmill Test to Detect Stress Induced Ischemic Heart Disease in Type 2 Diabetes Mellitus Patients Asymptomatic for CAD: A Hospital Based Cross-sectional Study in Rural Population of Central India. J Diabetes Metab 4: 244. doi:10.4172/2155-6156.1000244

Page 3 of 5

\section{Risk factors associated with silent ischemia}

The impact of lipid parameters and micro vascular complications on TMT outcome was evaluated in terms of odds ratio as shown in table 2. For continuous variables, the unadjusted odds ratios were obtained through univariate logistic regression. Adjusted odds ratios were obtained through multivariate logistic regression by including covariates that were significant in bivariate analysis. Accordingly, age, duration of diabetes and family history of IHD, along with the risk factor of interest were included and the models were obtained.

Glycemic control $\left(\mathrm{HbA}_{1} \mathrm{C}\right)$ showed increased risk of inducible ischemia with adjusted odds ratio of 1.341 (95\% CI: 1.055-1.734), which was statistically significant with $\mathrm{P}$ value 0.019 . However, for other lipid parameters, the risk of inducible ischemia remained unchanged as indicated by odds ratio values closed to 1.0. The ratio LDL/HDL showed increased risk of inducible ischemia with adjusted odds ratio of 2.097 (95\% CI: 1.151-4.207), which was statistically significant with
$\mathrm{P}$ value 0.023 . Also, the ratio TC/HDL significantly increased adjusted odds $(2.251 ; 95 \%$ CI: $1.416-3.770)$ in favor of inducible ischemia (P value $=0.002$ )

As regards micro vascular complications, Nephropathy showed significant impact on risk of inducible ischemia. Table 2 shows that with the change in the level from nil to micro, the odds for inducible ischemia increases 2.188 (95\% CI: 0.794-6.028) times; while the change of level from nil to macro increases the odds 7.977 (95\% CI: 1.348 47.202) times in favor of inducible ischemia. Presence of retinopathy also indicated significant increase in the risk of inducible ischemia with adjusted OR of 2.807 (95\% CI: 1.037-7.595). However, neuropathy did not show any impact on TMT positive result as indicated by adjusted odds ratio close to $1(0.987 ; 95 \%$ CI: 0.802-1.275).

\section{Discussion}

Diabetes Mellitus appears to confer a dramatic increase in the risk of silent ischemia with most studies suggesting a prevalence of $10-20 \%$

\begin{tabular}{|c|c|c|c|c|}
\hline \multirow[b]{2}{*}{ Characteristic } & \multirow[b]{2}{*}{ Category } & \multicolumn{3}{|c|}{ TMT result } \\
\hline & & Positive $(n=34)$ & Negative $(n=90)$ & Inconclusive $(n=37)$ \\
\hline Age $(\mathrm{yrs})^{*} \dagger($ Mean $\pm \mathrm{SD})$ & & $53.56 \pm 7.41$ & $48.71 \pm 8.72$ & $48.51 \pm 8.48$ \\
\hline \multirow[t]{2}{*}{$\operatorname{Sex}(\%)$} & Male & $19(55.88 \%)$ & $60(66.67 \%)$ & $18(48.65 \%)$ \\
\hline & Female & $15(44.12 \%)$ & $30(33.33 \%)$ & $19(51.35 \%)$ \\
\hline Duration of diabetes (months) ${ }^{*}($ Mean \pm SD) & & $74.79 \pm 48.2$ & $47.41 \pm 31.66$ & $58.66 \pm 39.22$ \\
\hline \multirow[t]{2}{*}{ Family history $\ddagger(\%)$} & None & $17(50 \%)$ & $67(74.44 \%)$ & $29(78.38 \%)$ \\
\hline & IHD & $17(50 \%)$ & $23(25.55 \%)$ & $8(21.62 \%)$ \\
\hline \multirow[t]{2}{*}{ Personal history (\%) } & None & $23(67.65 \%)$ & $64(71.11 \%)$ & $27(72.97 \%)$ \\
\hline & Smoker/Alcoholic/Both & $11(32.35 \%)$ & $26(28.89 \%)$ & $10(27.02 \%)$ \\
\hline \multirow[t]{2}{*}{ Diet $(\%)$} & Vegetarian & $6(17.65 \%)$ & $21(23.33 \%)$ & $7(18.92 \%)$ \\
\hline & Mixed & $28(82.35 \%)$ & $69(76.67 \%)$ & $30(81.08 \%)$ \\
\hline \multirow[t]{3}{*}{ Activity (\%) } & Sedentary & $14(41.18 \%)$ & $35(38.89 \%)$ & $16(43.24 \%)$ \\
\hline & Moderate & $18(52.94 \%)$ & $42(46.67 \%)$ & $15(40.54 \%)$ \\
\hline & Heavy & $2(5.88 \%)$ & $13(14.44 \%)$ & $6(16.22 \%)$ \\
\hline BMI $\left(\mathrm{Kg} / \mathrm{m}^{2}\right)($ Mean $\pm \mathrm{SD})$ & & $25.62 \pm 4.21$ & $24.33 \pm 4.34$ & $24.46 \pm 4.36$ \\
\hline
\end{tabular}

${ }^{*}$ Comparison of characteristic between TMT positive and negative groups resulting into $P<0.05$ as per Student's $t$-test †Comparison of characteristic between TMT positive and inconclusive groups resulting into $P<0.05$ as per Student's $t$-test ¥Comparison of characteristic between TMT positive and negative groups resulting into $P<0.05$ as per Chi-square test

Table 1: Descriptive statistics for general characteristics of study population according to TMT results $(n=161)$

\begin{tabular}{|c|c|c|c|c|c|}
\hline \multirow[b]{2}{*}{ Variable } & \multirow[b]{2}{*}{ Category } & \multicolumn{2}{|c|}{ Unadjusted Odds ratio } & \multicolumn{2}{|c|}{ Adjusted Odds ratio* } \\
\hline & & OR $(95 \% \mathrm{Cl})$ & $P$ value & OR $(95 \% \mathrm{Cl})$ & $P$ value \\
\hline \multicolumn{6}{|l|}{ Bio-chemical } \\
\hline $\mathrm{HbA}_{1} \mathrm{C}$ & & $1.482(1.155-1.901)$ & 0.002 & $1.341(1.055-1.734)$ & 0.019 \\
\hline TC & & $1.024(1.010-1.039)$ & 0.001 & $1.024(1.011-1.039)$ & 0.001 \\
\hline TG & & $1.013(1.006-1.020)$ & $<0.0001$ & $1.015(1.008-1.023)$ & $<0.0001$ \\
\hline LDL & & $1.018(1.000-1.036)$ & 0.044 & $1.024(1.005-1.045)$ & 0.018 \\
\hline HDL & & $0.996(0.949-1.047)$ & 0.888 & $1.000(0.949-1.047)$ & 0.863 \\
\hline LDL/HDL & & $1.681(0.933-3.027)$ & 0.084 & $2.097(1.151-4.207)$ & 0.023 \\
\hline TC/HDL & & $2.135(1.323-3.444)$ & 0.002 & $2.251(1.416-3.770)$ & 0.002 \\
\hline VLDL & & $1.024(0.982-1.068)$ & 0.272 & $1.027(0.984-1.074)$ & 0.207 \\
\hline \multicolumn{6}{|l|}{ Nephropathy } \\
\hline & Nil & 1 & \multirow{3}{*}{0.001} & 1 & \multirow{3}{*}{0.042} \\
\hline & Micro & $2.905(1.166-7.241)$ & & $2.188(0.794-6.028)$ & \\
\hline & Macro & $16.100(3.038-85.331)$ & & $7.977(1.348-47.202)$ & \\
\hline \multirow[t]{2}{*}{ Retinopathy } & No & 1 & \multirow{2}{*}{0.018} & 1 & \multirow[b]{2}{*}{0.042} \\
\hline & Yes & $2.800(1.189-6.592)$ & & $2.807(1.037-7.595)$ & \\
\hline \multirow[t]{2}{*}{ Neuropathy } & No & 1 & \multirow{2}{*}{0.325} & 1 & \multirow[b]{2}{*}{0.421} \\
\hline & Yes & $1.002[0.892-1.221]$ & & 0.987 [0.802-1.275] & \\
\hline
\end{tabular}

*Odds ratio obtained after adjusting for age, duration of diabetes and family history

Table 2: Odds ratio associated with risk factors of IHD. 
Citation: Lavekar AS, Salkar HR (2013) Treadmill Test to Detect Stress Induced Ischemic Heart Disease in Type 2 Diabetes Mellitus Patients Asymptomatic for CAD: A Hospital Based Cross-sectional Study in Rural Population of Central India. J Diabetes Metab 4: 244. doi:10.4172/2155-6156.1000244

[5]. Type $2 \mathrm{DM}$ is a Framingham risk factor for coronary arterial diseases (CAD) [4]. Diagnosis of CAD in type $2 \mathrm{DM}$ patients is largely based on the recommendations of American Diabetic Association (ADA), which advocates use of TMT or coronary arterial angiography for diagnosis of silent ischemia [6]. Hyperglycemia causes vascular disturbances especially endothelial dysfunction contributed by abnormal nitric oxide, increased endothelium, angiotensin II and reduced prostacyclin activity. Diabetic dyslipidemia further contributes to atherosclerotic risk, which is mainly due to increased LDL and decreased HDL. The vascular effects of the advanced glycosylated end products (AGEs), circulating fatty acids aggravate the systemic inflammation due to oxidative stress [7]. Smoking, hypertensions, dyslipidemia, family history of CAD and micro and macro albuminuria are the proven risk factors for CAD in type $2 \mathrm{DM}$ patients [6]. Duration of diabetes is not a proven risk factor for silent ischemia according to ADA guidelines. However, there are several studies reporting positive correlation between the two [8-10]. Our study also supports the finding that duration is a strong predictor of IHD. There are studies recommending routine screening for IHD with TMT for patients who are suffering from DM for more than 10 years [8]. Gender as a risk factor to silent ischemia is insignificantly associated with TMT result, and this is in unison with the study done by Gulam et al. [11]. Statistically significant correlations between increased TG levels, increased TC levels and decreased HDL levels with coronary arterial disease have already been established $[9,12]$. However, some contrasting results were obtained from the DIAD study and several others showing that there is hardly any correlation of dyslipidemia with silent ischemia $[13,14]$. In this study, we found TC, TG, LDL/HDL ratio and TC/HDL ratio as significantly associated with silent ischemia. Role of glycemic control as a predictor of silent ischemia is yet to be established [15]. However, this study reveals that poor glycemic control and silent ischemia are significantly associated with each other. Micro and macro albumin are the proven risk factors for CAD. This study also confirms the same and resulted into sensationally high association of macroalbuminuria with increased risk of inducible ischemia. Some other studies also have shown the same results [16]. Whether diabetic retinopathy is associated with IHD or not is a matter of debate; however, few studies have reported retinopathy as predictor of IHD $[17,18]$. Through this study, we found a positive association between the two. Even though, autonomic dysfunction is strongly associated with IHD in DM patients, existence of peripheral neuropathy as a predictor of IHD is still not clear. Our study did not show any significant association between neuropathy and silent ischemia.

Stress echo, as a screening tool for diagnosis of IHD is not recommended by ADA. It should be done only in cases when there is high probability of false positive TMT results. A negative stress echo implies that the likelihood of patient having coronary artery disease (CAD) is low [19]. Considering this, stress echo was performed only in TMT positive cases in our study.

The most common reason for inconclusive TMT result was the inability to complete TMT due to fatigue. Other reasons included, arthritic pain, inability to achieve targeted heart rate despite good exercise tolerance, hypersensitive BP response during TMT and fear of performing TMT.

In conclusion, proportion of silent ischemia in type $2 \mathrm{DM}$ patients from rural population of Central India was estimated to be $21.11 \%$ based on TMT. Age, duration of DM and family history of DM / IHD were identified as significant covariates. Total cholesterol, Triglycerides, LDL/HDL, TC/HDL, poor glycemic control, diabetic nephropathy and retinopathy were the key predictors of silent ischemia in patients who underwent TMT. The study revealed that TMT has nearly $74 \%$ accuracy of predicting CAD and could be a simple, easily available, inexpensive and non-invasive test in rural settings, even for screening purpose. Undoubtedly, tools such as MDCT, coronary angiography are more promising in detection of silent ischaemia with good accuracy [4]. However, the problem with these tools is their unavailability and applicability in rural / remote areas. Thus, we believe that TMT as a screening method followed by stress echo can be an effective means of diagnosis of asymptomatic CAD in type $2 \mathrm{DM}$ patients in such rural set ups.

\section{Acknowledgements}

The authors would like to thank Dr. Dhananjay Raje, Head, Data Analysis Group, MDS Bio-Analytics Pvt. Ltd. Nagpur, India for statistical analysis support and reviewing the manuscript.

\section{References}

1. Ali MK, Narayan KM, Tandon N (2010) Diabetes and coronary heart disease: Current perspectives. Indian J Med Res 132: 584-597.

2. Alexander CM, Landsman PB, Teutsch SM (2000) Diabetes mellitus, Impaired fasting glucose, atherosclerotic risk factors, and prevalence of coronary heart disease. AM J Cardiol 86: 897-902.

3. American Diabetes Association (2010) Standards of medical care in diabetes--2010. Diabetes care 33: S11-S61.

4. Yoo WS, Kim HJ, Kim D, Lee MY, Chung HK (2009) Early Detection of Asymptomatic coronary artery disease in patients with type 2 Diabetes Mellitus. Korean J Intern Med 24: 183-189.

5. Naka M, Hiramatsu K, Aizawa T, Momose A, Yoshizawa K, et al. (1992) Silent myocardial ischemia in patients with non-insulin-dependent diabetes mellitus as judged by treadmill exercise testing and coronary angiography. Am Heart J 123: 46-53.

6. (1998) Consensus development conference on the diagnosis of coronary heart disease in people with diabetes: 10-11 February 1998, Miami, Florida. American Diabetes Association. Diabetes care 21: 1551-159.

7. Chopra S, Peter S (2012) Screening for coronary artery disease in patients with type 2 diabetes mellitus: An evidence-based review. Indian J Endocrino Metab 6: 94-101

8. Janand-Delenne B, Savin B, Habib G, Bory M, Vague P, et al. (1999) Silent myocardial ischemia in patients with diabetes: who to screen. Diabetes Care 22: $1396-1400$.

9. (1997) Prevalence of unrecognized silent myocardial ischemia and its association with risk factors in non insulin dependent diabetes mellitus. Milan study on Atherosclerosis and Diabetes Group. Am J Cardiol 79: 134-139.

10. Langer A, Freeman MR, Josse RG, Steiner G, Armstrong PW (1991) Detection of silent myocardial ischemia in diabetes mellitus. Am J Cardiol 67: 1073-1078.

11. Ghulam HB, Shaikh S, Syed Zulfiquar SA, Devrajani BR, Rajpar N, et al. (2011) Silent ischemia in patients with type 2 Diabetes Mellitus. World Applied Sciences 13: 213-216

12. Gazzaruso C, Garzaniti A, Giordanetti S, Falcone C, Fratino P (2002) Silen coronary Artery disease in type 2 Diabetes mellitus: The role of lipoprotein(a) homocysteine and apo(a) polymorphism. Cardiovasc Diabetology 1: 5 .

13. Bacci S, Villella M, Villella A, Langialonga T, Grilli M, et al. (2002) Screening of silent myocardial ischemia in type 2 diabetic patients with additional atherogenic risk factors: applicability and accuracy of the exercise stress test. Eur J Endocrinol 147: 649-654

14. Wackers FJ, Young LH, Inzucchi SE, Chyun DA, Davey JA, et al. (2004) Detection of silent myocardial ischemia in asymptomatic diabetic subjects: the DIAD study. Diabetes care 27: 1954-1961.

15. Sargin H, Ozisik M, Ozisik NC, Omer S, Orbay E, et al. (2005) The Prevalence of silent ischemia in Turkish Patients with type 2 diabetes mellitus. Tohok J Exp Med 205: 351-355.

16. Foster MC, Hwang SJ, Larson MG, Parikh NI, Meigs JB, et al. (2007) Cross- 
Citation: Lavekar AS, Salkar HR (2013) Treadmill Test to Detect Stress Induced Ischemic Heart Disease in Type 2 Diabetes Mellitus Patients Asymptomatic for CAD: A Hospital Based Cross-sectional Study in Rural Population of Central India. J Diabetes Metab 4: 244. doi:10.4172/2155-6156.1000244

Page 5 of 5

classification of microalbuminuria and reduced glomerular filtration rate: associations between cardiovascular disease risk factors and clinical outcomes. Arch Inter Med 167: 1386-1392.

17. Hernández C, Candell-Riera J, Ciudin A, Francisco G, Aguadé-Bruix S, et al. (2011) Prevalence and risk factors accounting for true silent myocardial ischemia: a pilot case-control study comparing type 2 diabetic with non-diabetic control subjects. Cardiovasc Diabetol 10: 9.
18. Forood A, Majomi M (2008) Risk Factors for Silent Myocardial Ischaemia in Type II Diabetic Patients. Iranian Heart Journal 9: 37-42.

19. ACC/AHA guidelines for clinical application of echocardiography (1997) A Report of the American College of Cardiology/ American Heart Association Task Force on Practice Guidelines (Committee on Clinical Application of Echocardiography) Developed in Collaboration With the American Society of Echocardiography. Circulation 95: 1686-1744. 